\title{
The wear characteristics of a high power diode laser generated glaze on the ordinary Portland cement surface of concrete
}

\begin{abstract}
J. Lawrence and L. Li
Laser Process Engineering Group, Manufacturing Division, Department of Mechanical Engineering, University of Manchester Institute of Science and Technology (UMIST), Manchester, M60 1QD, UK.
\end{abstract}

\section{Correspondence}

Dr. Jonathan Lawrence

Manufacturing Division,

Department of Mechanical Engineering,

University of Manchester Institute of Science and Technology (UMIST),

Manchester,

M60-1QD,

UK.

Tel : (44) 1612363311 ext. 2383

Fax : (44) 1612003803

email : j.lawrence@stud.umist.ac.uk 


\begin{abstract}
The ordinary Portland cement (OPC) surface layer of concrete, which was glazed using a high power diode laser (HPDL), has been tested in order to determine the wear characteristics of the glaze. The work showed that the generation of a surface glaze resulted in the considerable enhancement of the wear characteristics over an untreated OPC surface of concrete. Within both normal and corrosive (detergent, $\mathrm{NaOH}$ and $\mathrm{HNO}_{3}$ ) environmental conditions the wear rate of the HPDL generated glaze was $3.5 \mathrm{mg} \cdot \mathrm{cm}^{-2} \cdot \mathrm{h}^{-1}$. In contrast, the untreated OPC surface of concrete exhibited a wear rate of 9.8 $114.8 \mathrm{mg} \cdot \mathrm{cm}^{-2} \cdot \mathrm{h}^{-1}$ when exposed to the various reagents. Life assessment testing revealed that the laser glazed OPC surfaces effected an increase in actual wear life of 1.3 to 17.7 times over the untreated OPC surface of concrete, depending upon the corrosive environment. The reasons for these marked improvements in the wear resistance and wear life of the HPDL generated glaze over the untreated OPC surface of concrete can be attributed to firstly, the vitrification of the OPC surface after HPDL treatment which subsequently created a much more dense and consolidated surface, and secondly, the generation of a surface with improved microstructure and phase which is more resistant in corrosive environments.
\end{abstract}

Keywords: High power diode laser (HPDL); Concrete; Ordinary Portland cement; Surface glazing; Wear; Life characteristics 


\section{Introduction}

The economic implications of wear are a cause of considerable concern to building and civil engineers alike who employ concrete in a broad area of applications. Furthermore, in the present and foreseeable world economy, material conservation is becoming a matter of increasing importance. Wear is a major cause of concrete wastage in many application areas where over time and as a direct consequence of the operating environment, the concrete becomes corroded and/or contaminated. This ultimately necessitates the arduous and costly undertaking of repairing or replacing the affected concrete either by physical or mechanical means. Clearly, any means by which the life of the concrete could be extended would be of great interest to all engineers, since any reduction in concrete wear could effect significant economic savings.

Owing to their unique characteristics, lasers have the propensity to be employed for the non-contact processing of materials, such as concrete, which are otherwise difficult to process. To date, many studies have been carried out to investigate the laser processing of concrete. Most of the research, however, has concentrated on the laser cutting of concrete and reinforced concrete using high power $\mathrm{CO}_{2}$ lasers, most prominently with regard to nuclear reactor decommissioning [1-3]. Also, as part of nuclear plant decommissioning, Li et al. [4-7] conducted research to determine the workability of several laser techniques for sealing/fixing radioactive contamination onto concrete surfaces. Such techniques experimented with were: direct glazing of the concrete, single and multiple layer fusion cladding and combined chemical/fusion cladding. Work by Sugimoto et al. [8] focused upon modifying the surface appearance and surface properties of cement based materials using a high power $\mathrm{CO}_{2}$ laser. The laser treatment produced novel surfaces, with surface textures, properties and appearance unique to laser treatment. The resultant physical characteristics and mechanical behaviour of the post-process cement based materials was later fully characterised by Wignarajah et al. [9]. Borodina et al. [10] has carried out investigations into the structural changes within the composition of zirconia concrete caused by surface exposure to $\mathrm{CO}_{2}$ laser radiation, detailing microstructural changes, phase changes and the absorptivity characteristics. In all of these studies, spallation and excessive cracking and porosity formation were found to be major problems undermining the performance of the laser treated surface layer. 
In contrast, more detailed and comprehensive studies conducted by Lawrence and Li have investigated the feasibility and characteristics of high power diode laser (HPDL) generated glazes on the ordinary Portland cement (OPC) surface of concrete $[11,12]$. In further work Lawrence and Li studied the generation and carried out a comparison of the glazes generated with both $\mathrm{CO}_{2}$ and HPDL's $[13,14]$.

This present work is concerned with establishing the wear characteristics of a glaze generated on the OPC surface layer of concrete by means of a HPDL. This is of particular interest since the inherent compactness of the HPDL enables it to be portable. Other industrial lasers only allow the workpiece to be processed at a dedicated laser work station, whereas on the other hand, the HPDL can be used for on-site processing. Thus the of the HPDL for the processing of building materials may lead to many new practical applications.

\section{Experimental procedures}

\subsection{Materials}

The concrete studied in the experiments was the ubiquitous OPC based concrete, with the usual 'as cast' OPC surface of concrete being the subject of the HPDL irradiation. The OPC surface of the concrete had a thickness of $2.5 \mathrm{~mm}$. For the purpose of experimental convenience the as-received concrete blocks were sectioned into squares $(120 \times 120 \times 20 \mathrm{~mm})$ prior to laser treatment. The composition of the concrete bulk by volume is as follows: $20 \mathrm{~mm}$ limestone aggregate $(40 \%), 10 \mathrm{~mm}$ limestone aggregate (14\%), zone M sand (28.5\%), OPC (10.5\%) and particulate fine aggregate (7\%).

\subsection{Laser processing procedure}

The laser used in the study was a surgical HPDL (Diomed Ltd.), emitting at $810 \mathrm{~nm} \pm 20 \mathrm{~nm}$ and operating in the $\mathrm{CW}$ mode with rated optical powers ranging from 0-60 W. The laser beam was delivered to the work area by means of a $4 \mathrm{~m}$ long, $600 \mu \mathrm{m}$ core diameter optical fibre, the end of which was connected to a 2:1 focusing lens assembly mounted on the z-axis of a 3-axis CNC gantry table. The concrete sample blocks were irradiated using the defocused high order mode HPDL beam with a beam spot diameter of $2 \mathrm{~mm}$ and laser powers (measured at the workpiece using a Power Wizard power meter) of 20-55 W. As shown in Fig. 1, the defocused laser beam was fired across the 
surfaces of the concrete samples by traversing the samples beneath the beam using the $\mathrm{x}$ - and $\mathrm{y}$-axis of the CNC gantry table at speeds ranging from $60-600 \mathrm{~mm} / \mathrm{min}$. Area coverage of the OPC surface of the concrete was achieved by overlapping the glaze tracks. The overlap was $0.3 \mathrm{~mm}$. The laser optics were protected by means of a coaxially blown $\mathrm{O}_{2}$ shield gas jet a rate of $5 \mathrm{l} / \mathrm{min}$.

As detailed comprehensively by Lawrence and Li $[11,12]$, HPDL interaction with the OPC surface of concrete effected the vitrification of the surface, resulting in the generation of a glaze of around $750 \mu \mathrm{m}$ in thickness. The surface of the glazes typically exhibited an undulating morphology, with the undulations being regular in both periodicity and intensity. In addition, the surface displayed very few microcracks and few porosities. As with any laser surface treatment process, HPDL treatment of the OPC surface of concrete resulted in the generation of an attendant heat affected zone (HAZ). In previous work conducted by Lawrence and Li [11-14] this HAZ was identified as being comprised of unslaked lime $(\mathrm{CaO})$, the formation of which was due to the dehydration of the OPC as a result of the heat generated when the HPDL beam was incident with the OPC surface.

\subsection{Wear testing procedure}

To determine the wear resistance characteristics of the HPDL generated OPC glaze and also those of an untreated OPC surface, wear tests were conducted in accordance with Fig. 2. For experimental purposes the concrete was cut into smaller pieces $(25 \mathrm{~mm}$ x $25 \mathrm{~mm}$ ). Half of the samples were then laser treated. All the samples were then weighed. The samples were then clamped individually in the vice of a common shaping machine. A steel abrader was attached to the floating head of the shaping machine and moved cyclically back and forth across the untreated and HPDL glazed OPC surfaces. The total distance moved in one cycle was $6 \mathrm{~mm}$ while the traverse speed was $180 \mathrm{~mm} / \mathrm{min}$. By applying weights to the floating head a frictional force of $60 \mathrm{~N}$ was generated. The samples were subjected to the frictional force for 8 hours, being removed from the machine and weighed at two hourly intervals.

\section{Results}




\subsection{Wear characteristics in normal environmental conditions}

Generally, the wear resistance of any material is primarily related to the hardness of the material in comparison with the that of other materials with which it subsequently comes into contact with [15]. However, wear resistance is not directly proportional to hardness, nor does it always increase with hardness [16]. Fig. 3 shows the relationship between weight loss and the friction time for HPDL generated OPC glazes and the untreated OPC surfaces. As is evident from Fig. 3, the wear resistance of the HPDL generated OPC glaze displays a significant increase in wear resistance over the untreated OPC surface, with the weight loss being approximately 2 times lower after 4 hours, and 3 times lower after 8 hours.

\subsection{Wear characteristics in corrosive environments}

Concrete surfaces are often subjected to corrosive substances, either as part of the normal service environment and/or as a result of routine cleaning. Therefore corrosion resistance tests based upon BS 6431 [17] were conducted using nitric acid, sodium hydroxide and Premier Products MP9 detergent cleaner. The experiments were carried out by dropping small amounts of the corrosive agents, in the concentration ratios of $80 \%, 60 \%, 40 \%, 20 \%$ and $10 \%$, on to the untreated and HPDL glazed OPC surfaces at hourly intervals for four hours. The samples were then examined optically and mechanically tested in terms of compressive strength and wear. High concentrations of the various corrosive agents were used principally to accelerate the tests. However, in practice $60 \%$ nitric acid is used within the nuclear processing industry as a solvent for nuclear fuels [18].

All three reagents in the concentrations $80 \%, 60 \%$ and $40 \%$ were seen to immediately attack the untreated OPC surface, with the nitric acid and sodium hydroxide attacking with greater severity than the detergent. On the other hand, the HPDL glazed OPC surfaces exhibited no discernible changes in either morphology or microstructure. In addition, no signs of devitrification due to corrosion were discernible.

Tests conducted according to ASTM C579-91 [19] revealed that exposure of the untreated OPC surface to the reagents had a significant effect on the compressive strength and the wear resistance of the OPC. As one can see from Fig. 4, exposure of the OPC to nitric acid and sodium hydroxide in the concentrations $40-80 \%$ resulted in an average loss of compressive strength of approximately $19-37 \%$. 
In the case of the detergent a discernible loss in compressive strength only occurred with concentrations above $40 \%$. Here the average loss in compressive strength for concentrations in the range $60-80 \%$ was approximately $17 \%$. This compares with no discernible difference in either the wear resistance or the compressive strength of the HPDL glazed OPC surfaces.

Fig. 5 shows the variation in wear resistance of the untreated OPC surface when exposed to the reagents with an $80 \%$ concentration. As one can see, the wear resistance is significantly affected, particularly through interaction with the nitric acid and the sodium hydroxide. Here the weight loss was approximately 5 times higher than for the unexposed OPC after 4 hours, and approximately 11 times higher after 8 hours for the nitric acid. In the case of the detergent the weight loss was marginal after both 4 and 8 hours.

\section{Discussion}

The results of the wear tests show clearly that the HPDL generated OPC glaze exhibited better wear resistance characteristics than the untreated OPC surface. In particular, a considerable improvement in wear rate was observed when the tests were conducted in corrosive environments. The marked difference in wear rate between the HPDL glazed and untreated OPC surface, in both normal and corrosive environments, can be ascribed entirely to the difference in structure of the two surfaces. Whereas the HPDL generated glaze is fully amorphous in nature (Fig. 6(b), the untreated OPC is comprised of a porous polycrystalline structure (Fig. 6(a). As such, HPDL beam interaction with the surface of the OPC has led to vitrification of the surface as Fig. 7 shows, creating a much more dense and consolidated surface. In addition, the HPDL generated glaze is considerably harder than the untreated OPC surface, a respective value of 6 compared to 2 on the Mohs scale. Consequently, these two improvements in the nature of the surface of the OPC will inherently afford the HPDL generated glaze better wear resistance over an untreated OPC surface. Furthermore, owing to the amorphous nature of the HPDL generated glaze, the glaze will naturally posses a greater resistance to corrosive reagents than the polycrystalline structure of the untreated surface of the OPC which is readily attacked by acids and alkalis [20]. Indeed, from a microscopic examination of the HPDL generated glaze, no signs of devitrification due to corrosion were discernible. 
It is believed that the wear rate of the HPDL generated glaze could be improved if the formation of porosities on the glaze surface could be substantially reduced, or even avoided altogether. As is evident from Fig. 8, blister type porosities on the surface of the HPDL generated glaze were ever present features of the glaze morphology. For all instances of porosity formation the mechanism behind their development is the consequence of gas escaping from within the melt and disrupting the surface [21]. With regard to the OPC glaze, the gas is likely to be $\mathrm{CO}_{2}$ [9]. If the laser energy density incident on the $\mathrm{OPC}$ is too low, then the generated $\mathrm{CO}_{2}$ can not escape from the molten OPC surface easily because of the high viscosity of the melt. As such, when the $\mathrm{CO}_{2}$ eventually does penetrate the melt surface, the resultant porosity is not filled by the flow of the melt; since the insufficient energy density is unable to maintain a high enough temperature for an adequate length of time and thus decrease the overall viscosity of the melt [22]. In this case the porosities formed are typically small and shallow, being regular in both periodicity and intensity. On the other hand, if the laser energy density incident on the OPC surface of the concrete is too high, then boiling of the surface may happen. At the same time an increase in $\mathrm{CO}_{2}$ formation may occur within the melt. These individual pockets of $\mathrm{CO}_{2}$ formation may combine and rise to surface of the melt. Once the energy density decreases (as the laser traverses away), then the additional $\mathrm{CO}_{2}$ will attempt to escape from the molten surface. However, the solidifying melt will prevent this, causing bubbles to form. The excessive $\mathrm{CO}_{2}$ gas pressure will firstly cause the bubbles to expand and ultimately rupture the walls of the bubbles, creating a sharp 'knife edge' porosity [3,9]. These types of porosity are usually large, deep and randomly spaced. Clearly, the physical characteristics of the knife-edge porosities makes them more susceptible to wear at a higher rate than the main body of the glaze. Thus, if knife-edge porosity generation could be avoided than improvements in the wear rate of the HPDL generated glaze could be realised.

The generally superior mechanical and chemical performance of the laser generated OPC glazes over the untreated OPC suggests that the life characteristics of the OPC glazes may also be superior to those of untreated OPC. Yet in any analysis of the wear life of the two materials, the in-situ relative thickness of the HPDL generated glaze and the untreated OPC layer on concrete must be considered in order to give a true interpretation of the actual life characteristics. This is particularly true when considering the wear resistance (with and without exposure to corrosive chemical agents). Consequently the increase in wear life can be given by 


$$
\text { Increase in wear life }=\frac{\text { Laser glaze wear life }}{\text { Untreated OPC wear life }}
$$

where,

$$
\text { Wear life }=\frac{\text { Density } . \text { Thickness }\left(\mathrm{mg} \cdot \mathrm{cm}^{-3} \cdot \mathrm{cm}\right)}{\text { Wear rate }\left(\mathrm{mg} \cdot \mathrm{cm}^{-2} \cdot \mathrm{h}^{-1}\right)}
$$

Table 1 summarises the wear rate details and the nominal life increase of the HPDL generated glaze over the untreated OPC surface. As is clearly evident from Table 1, the HPDL generated glaze effected an increase in actual life over the untreated OPC surface regardless of the environment. Further, it can be seen that the increase in actual life of the HPDL generated glaze over the untreated OPC surface varies considerably depending upon the working environment. Notwithstanding this, arguably the most common working environment for an OPC surface would involve some contact with at least detergent acids, therefore yielding significant economic savings since a HPDL glazed OPC surface lasts around 2.5-times longer than one which is unglazed.

\section{Conclusion}

The wear characteristics of a glaze generated on the ordinary Portland cement (OPC) surface of concrete was using a $60 \mathrm{~W}$-cw high power diode laser (HPDL) have been determined. Within both normal and corrosive (detergent, $\mathrm{NaOH}$ and $\mathrm{HNO}_{3}$ ) environmental conditions the wear rate of the HPDL generated glaze was $3.5 \mathrm{mg} \cdot \mathrm{cm}^{-2} \cdot \mathrm{h}^{-1}$. In contrast, the untreated OPC surface of concrete exhibited a wear rate of $9.8 \mathrm{mg} \cdot \mathrm{cm}^{-2} \cdot \mathrm{h}^{-1}$ in normal environmental conditions and $18.5,73.8$ and $114.8 \mathrm{mg} \cdot \mathrm{cm}^{-2} \cdot \mathrm{h}^{-1}$ when exposed to detergent, $\mathrm{NaOH}$ and $\mathrm{HNO}_{3}$ respectively. Life assessment testing revealed that the HPDL generated glaze had an increase in wear life of 1.3 to 14.8 times over an untreated OPC surface, depending upon the corrosive environment. It is believed that the economic and material benefits to be gained from the deployment of such an effective and efficient coating on OPC could be significant.

The reasons for these marked improvements in the wear resistance and wear life of the HPDL generated glaze over the untreated OPC surface of concrete can be attributed to:

i. The vitrification of the OPC surface after HPDL treatment which subsequently created a much more dense and consolidated surface. 
ii. The generation of a surface with improved microstructure and phase which is more resistant in corrosive environments. 


\section{References}

1. K. Sugita, M. Mori, T. Fujioka, Applications of lasers for cutting concrete, Concrete Eng. 24 1986 13-22.

2. M. Hamasaki, Experimental cutting of biological shield concrete using a laser, Proceedings of the International Symposium on Laser Processing, San Jose, CA., USA, 1987, vol. 852, pp. 158-167.

3. H. Yoshizawa, S. Wignarajah, H. Saito, Study on laser cutting of concrete, Trans. Japan Welding Soc. 201989 31-36.

4. L. Li, P.J. Modern, W.M. Steen, Laser fixing and sealing of radioactive contamination on concrete surfaces, Proceedings of LAMP '92, Nagaoka, Japan, 1992, pp. 843-848.

5. L. Li, W.M. Steen, P.J. Modern, Proceedings of ISLOE '93, Singapore, 1994, pp 25-30.

6. L. Li, W.M. Steen, P.J. Modern, J.T. Spencer, Proceedings of RECOD '94, London, UK, 1994, pp 24-28.

7. L. Li, W.M. Steen, P.J. Modern, J.T. Spencer, Proceedings of EUROPTO '94: Laser Materials Processing and Machining, Frankfurt, Germany, 1994, pp 84-95.

8. K. Sugimoto, S. Wignarajah, K. Nagasi, S. Yasu, Fundamental study on laser treatment of architectural materials, Proceedings of ICALEO '90, Boston, MA., USA, 1990, vol. 71, pp 302312.

9. S. Wignarajah, K. Sugimoto, K. Nagai, Effect of laser surface treatment on the physical characteristics and mechanical behaviour of cement base materials, Proceedings of ICALEO '92, Orlando, FL., USA, vol. 75, pp. 383-393.

10. T.I. Borodina, G.E. Valyano, N.I. Ibragimov, E.P. Pakhomov, A.I. Romanov, L.G. Smirnova, P.K. Khabibulaev, Compositional changes in structure of zirconia concrete after exposure to $\mathrm{CO}_{2}$ laser radiation, J. Phys. and Chem. of Mater. Treatment 251995 541-546.

11. J. Lawrence, L. Li, Surface glazing of concrete using a $2.5 \mathrm{~kW}$ high power diode laser and the effects of beam geometry, Optics and Laser Tech. 312000 583-591. 
12. J. Lawrence, L. Li, High power diode laser surface glazing of concrete, J. Laser Apps. 712000 18.

13. J. Lawrence, L. Li, Comparison of surface glaze characteristics of concrete treated with a $\mathrm{CO}_{2}$ laser and a high power diode laser, Part I: Glaze formation mechanisms and characteristics, Mater. Sci. Eng. A, 2842000 93-102.

14. J. Lawrence, L. Li, Comparison of surface glaze characteristics of concrete treated with a $\mathrm{CO}_{2}$ laser and a high power diode laser, Part II: Mechanical, chemical and physical properties, Mater. Sci. Eng. A, 2872000 25-29.

15. H. Dickson, Glass: A Handbook for Students and Technicians, Hutchinsons Scientific and Technical Publications, London, 1951, p 148.

16. A. Petitbon, L. Boquet, D. Delsart, Laser surface sealing and strengthening of zirconia coatings, Surf. \& Coat. Tech. 491991 57-61.

17. BS 6431: Part 19: 1984, Ceramic Wall and Floor Tiles: Method for Determination of Chemical Resistance. Glazed Tiles.

18. J.T. Spencer, Letter to Author, October 1997.

19. ASTM C597-91, Standards Test Method for Compressive Strength of Chemical-Resistant Mortars, Grouts, Monolithic Surfacings and Polymer Concretes.

20. V.V. Vargin, Technology of Enamels, MacLaren \& Sons, London, 1965, pp. 125-128.

21. M.H. Lewis, Glasses and Glass-Ceramics, Chapman \& Hall, London, 1989, pp. 88-95.

22. Z. Liu, PhD Thesis, University of Liverpool, 1991. 


\section{List of Figures}

Fig. 1. Schematic illustration of the experimental set-up for the HPDL treatment of the OPC surface of concrete.

Fig. 2. Schematic illustration of the experimental set-up for the wear tests.

Fig. 3. Relationship between weight loss and friction time for the untreated and HPDL generated glaze on the OPC surface of concrete.

Fig. 4. Variation in compressive strength of the untreated OPC with reagent type and reagent concentration.

Fig. 5. Relationship between weight loss and friction time for the untreated OPC with different reagent types at the maximum concentration $(80 \%)$.

Fig. 6. XRD analysis of the OPC surface (a) before laser treatment and (b) after HPDL treatment.

Fig. 7. Typical SEM cross-section view of HPDL generated glaze on the OPC surface of concrete. (2.25 $\mathrm{kW} \mathrm{cm}^{-2}$ power density, $240 \mathrm{~mm} \mathrm{~min}^{-1}$ traverse speed)

Fig. 8. Typical optical surface morphology of the HPDL generated glaze on the OPC surface of concrete. (2.25 $\mathrm{kW} \mathrm{cm}^{-2}$ power density, $240 \mathrm{~mm} \mathrm{~min}^{-1}$ traverse speed) 
Fig. 1

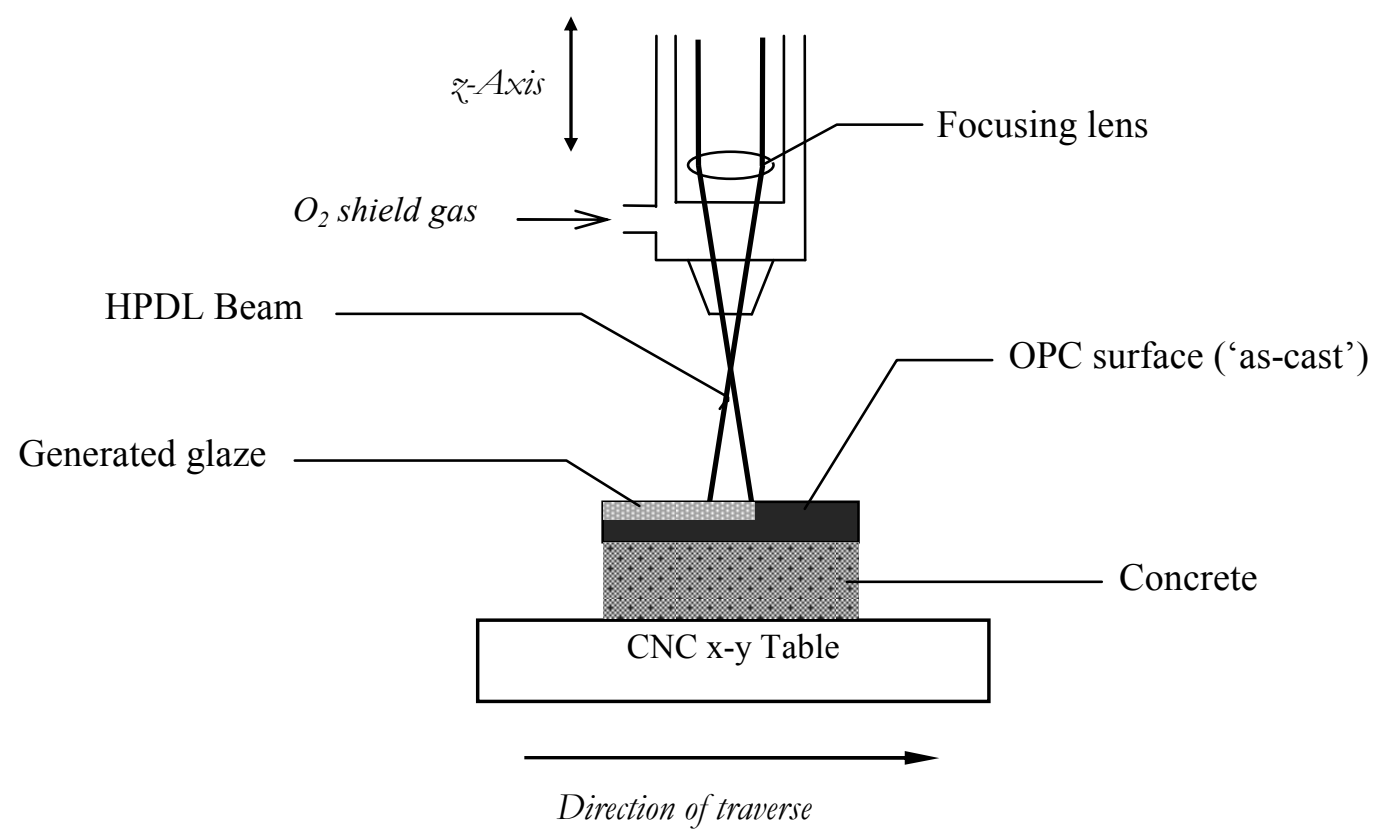


Fig. 2

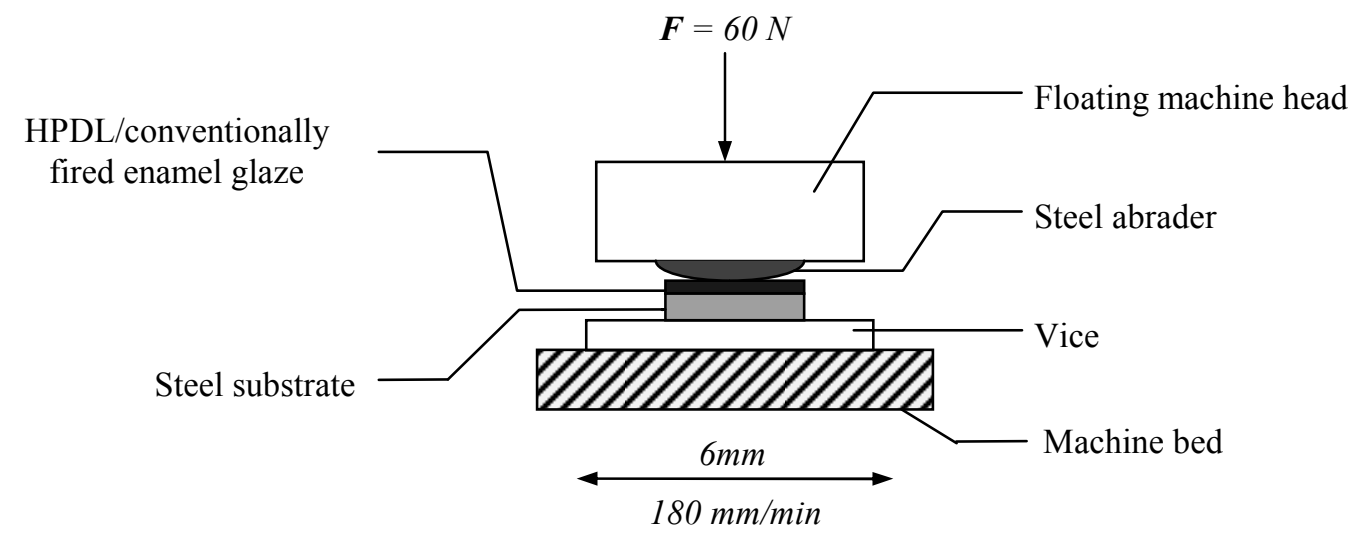


Fig. 3

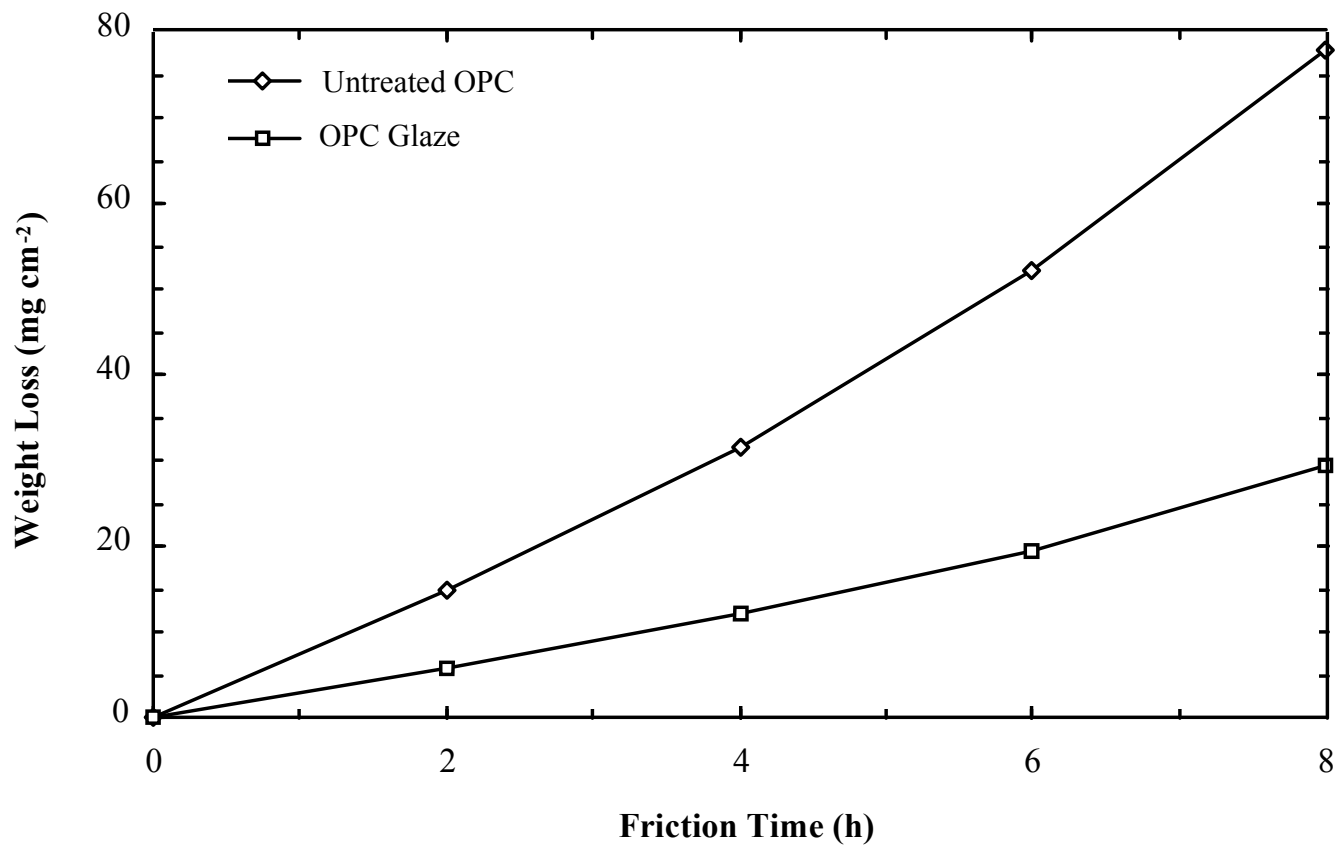


Fig. 4

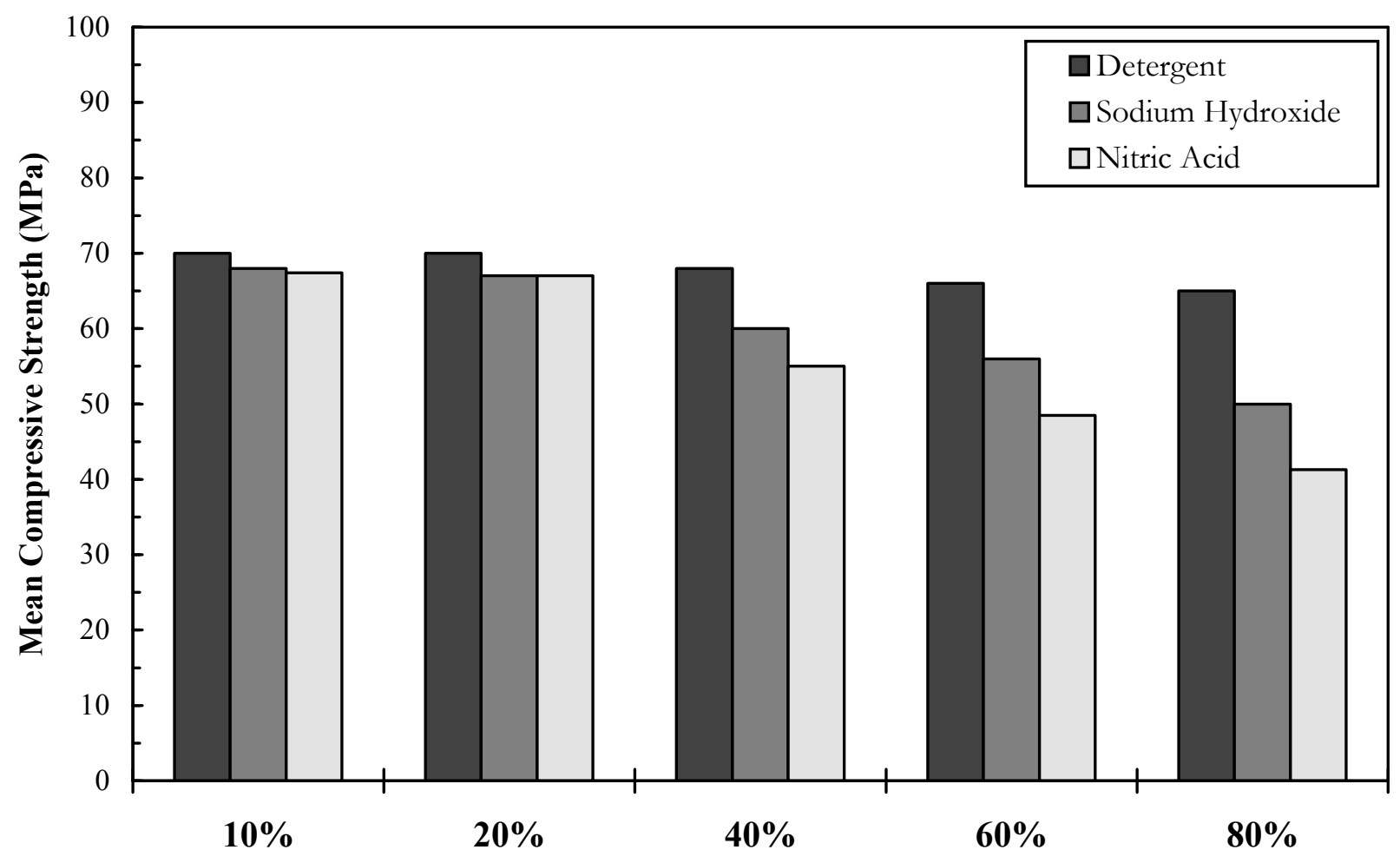


Fig. 5

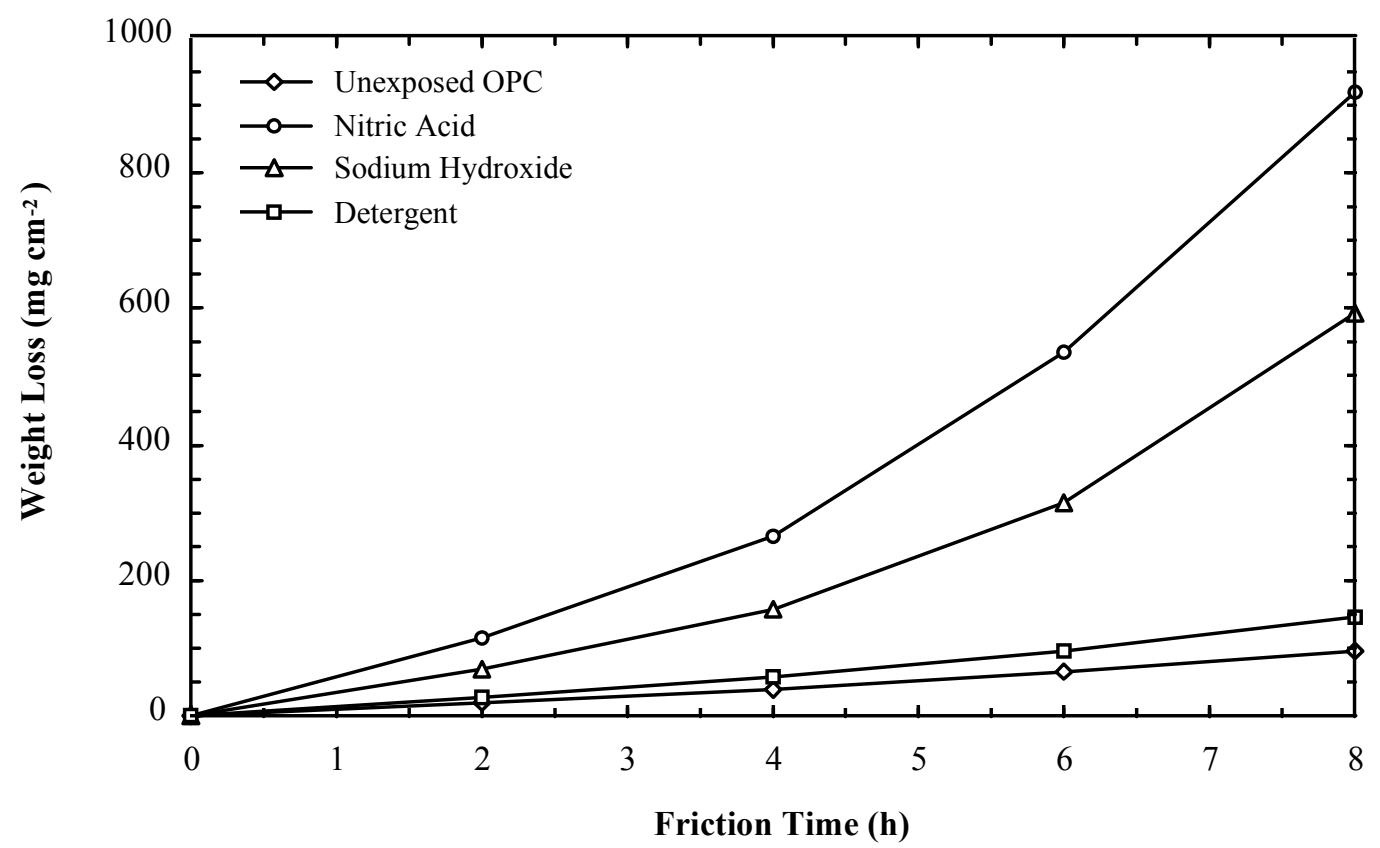


Fig. 6

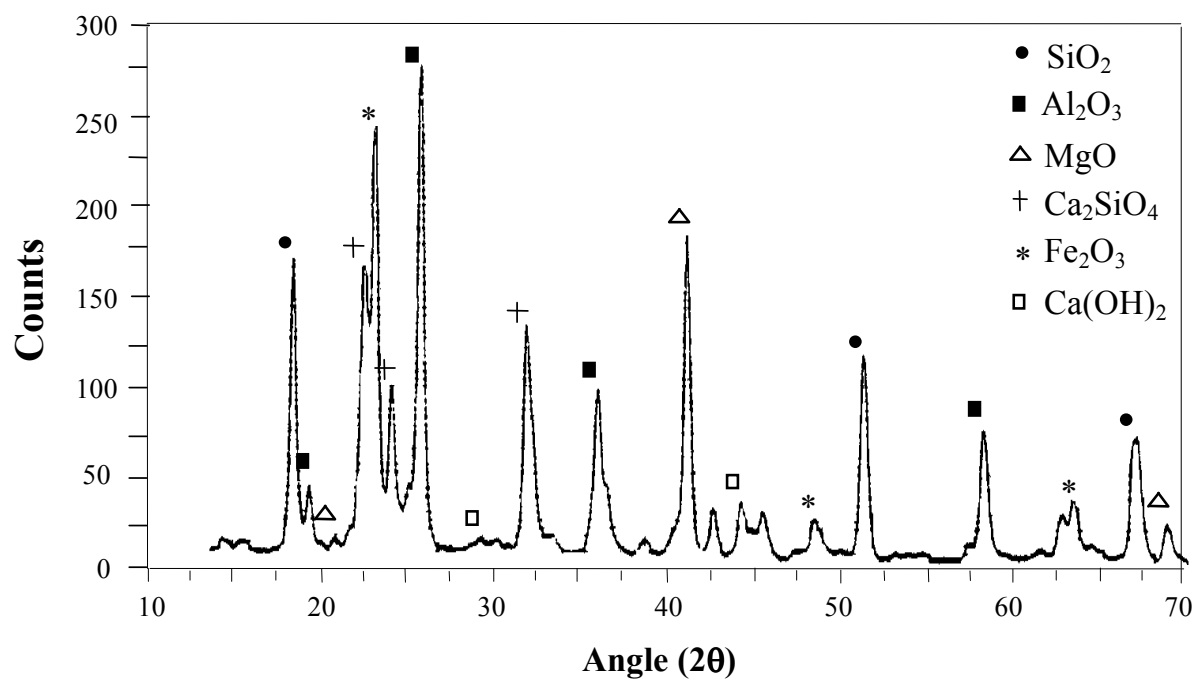

(a)

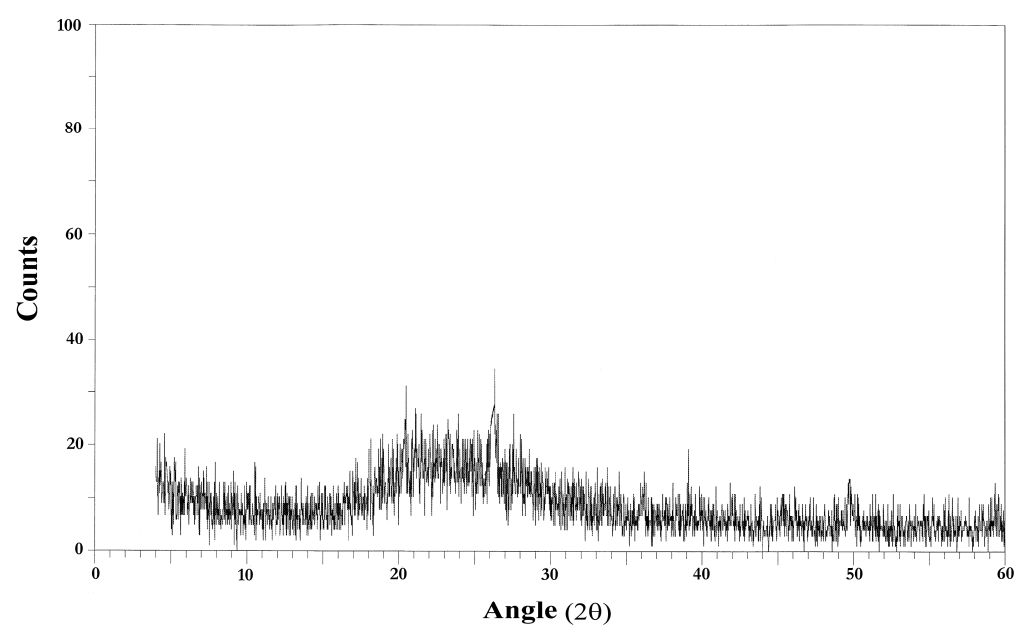

(b) 
Fig. 7

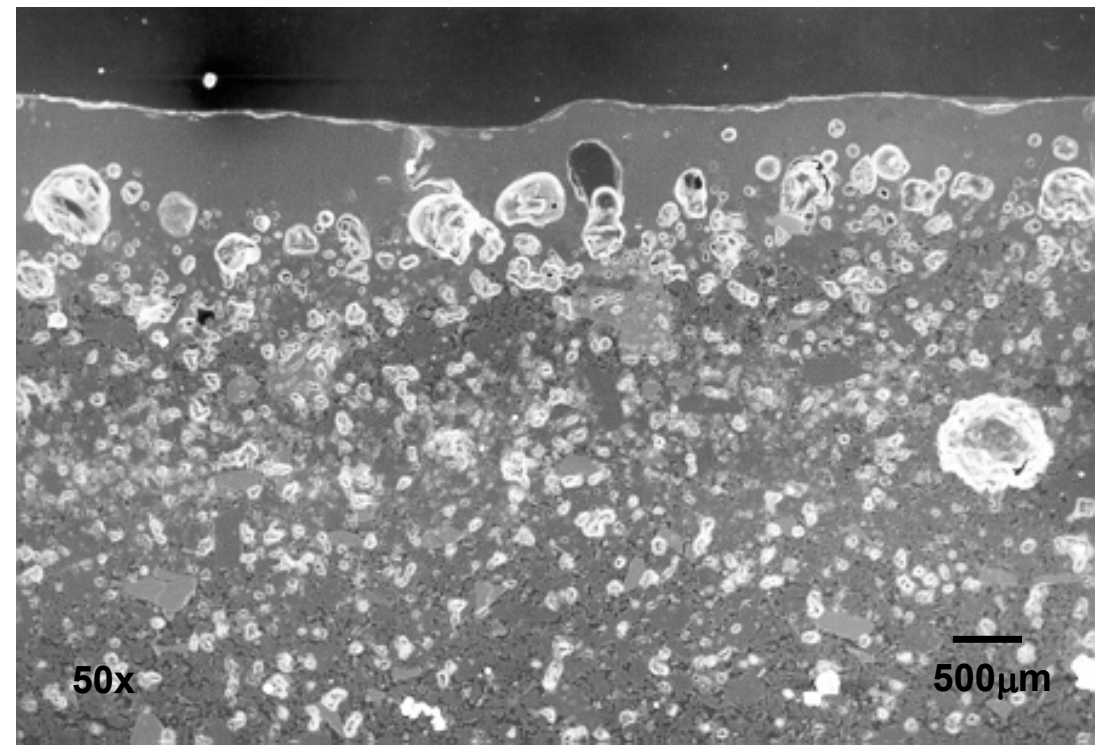


Fig. 8

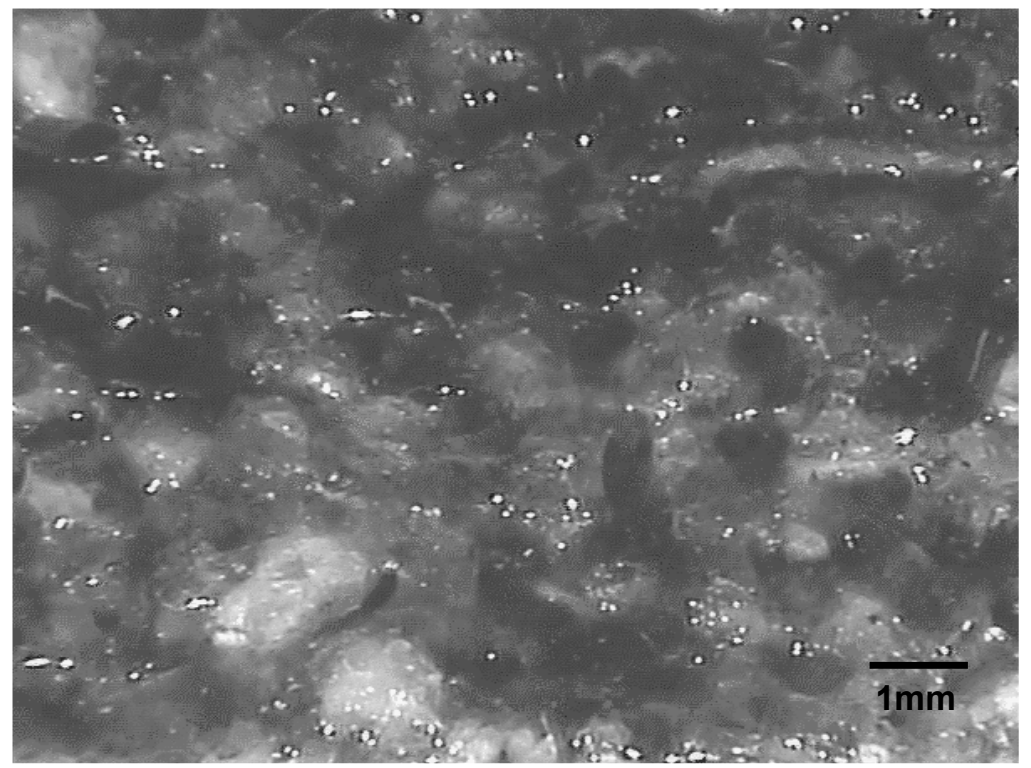




\section{List of tables}

Table 1. Wear rate details and the nominal life increase of the HPDL generated glaze over untreated $\mathrm{OPC}$ in various corrosive environments. 
Table 1.

\begin{tabular}{ccccccc}
\hline & & & \multicolumn{2}{c}{ Wear Rate $\left(\mathbf{m g . c m}^{-2} \cdot \mathbf{h}^{-1}\right)$} \\
& Density & Thickness & Unexposed & Detergent & NaOH & HNO $_{\mathbf{3}}$ \\
\hline Untreated OPC & $2220\left(\mathrm{~kg} \mathrm{~m}^{-3}\right)$ & $1500(\mu \mathrm{m})$ & 9.8 & 18.5 & 73.8 & 114.8 \\
HPDL OPC Glaze & $2000\left(\mathrm{~kg} \mathrm{~m}^{-3}\right)$ & $750(\mu \mathrm{m})$ & 3.5 & 3.5 & 3.5 & 3.5 \\
\hline Increase in Wear Life & $\sim$ & $\sim$ & $\mathbf{1 . 3}$ & $\mathbf{2 . 4}$ & $\mathbf{9 . 5}$ & $\mathbf{1 4 . 8}$ \\
\hline
\end{tabular}

